\title{
Inter-Spherulite Boundary Structure in Bulk-Crystallized Polyethylenes Directly Observed by Atomic Force Microscopy
}

\author{
Hongxiang TENG, Yi SHI, and Xigao JIN ${ }^{\dagger}$ \\ State Key Laboratory of Polymer Physics and Chemistry, Center for Molecular Science, Institute of Chemistry, \\ Chinese Academy of Sciences, Beijing 100080, China
}

(Received January 6, 2003; Accepted February 13, 2003)

\begin{abstract}
A regular structure formed between the spherulites crystallized from bulk polyethylene was observed directly by atomic force microscopy (AFM) for the first time. It was supposed that the regular structure originated from the stress field induced by the shrinkage effect of the polymeric bulk during its crystallization process. Also the regular structure provided a reasonable hypothesis about the crystallization process of macromolecules.
\end{abstract}

KEY WORDS Polyethylene / Spherulite / Structure / Atomic Force Microscopy (AFM) /

Generally, it is deemed that macromolecular crystals are formed by spherulites in micrometer scale, and spherulites are formed by lamellae in nanometer scale. Polymeric spherulites in micrometer scale have been studied adequately through polarized light microscopy (PLM) and scanning electron microscopy (SEM) in the past half century. ${ }^{1-4}$ It was concluded that the spherulite is formed by radiating lamellar fibrils along the radius direction. At the same time, macromolecular lamellae in nanometer scale have been investigated widely by transmission electron microscopy (TEM), electron diffraction (ED), and X-ray diffraction methods. ${ }^{5-7}$ It has been adopted that the macromolecular chains are approximately normal to the basal plane of the lamellae, parallel to the short direction. Comparatively, so far the structure between the spherulites of polymers has received less attention. Keith and Padden have carried out a series of work on the boundary structure between spherulites and lamellae by dissolving away the paraffin of small molecules that have been co-crystallized with polyethylene macromolecules. ${ }^{8,9}$ In the present paper, the morphological inter-spherulite boundary structure formed in a bulkcrystallized polyethylene thin film was observed directly by atomic force microscopy (AFM).

\section{EXPERIMENTAL}

The samples used in the experiment were a linear high-density polyethylene (HDPE) supplied by Beijing 2nd Reagent Factory, and a branched metallocene catalyzed linear low-density polyethylene (m-LLDPE) obtained from Exxon Co. The characteristic parameters of the linear and the branched polyethylenes were listed in Table I. The molecular weight (MW) and the

${ }^{\dagger}$ To whom correspondencd should be addressed.
Table I. Characterizations of the linear and branched polyethylenes used in this study

\begin{tabular}{cccccc}
\hline Samples & $M_{\mathrm{n}} \times 10^{-3}$ & $M_{\mathrm{w}} / M_{\mathrm{n}}$ & $\mathrm{SCB} / 1000 \mathrm{C}$ & $T_{\mathrm{m}}\left({ }^{\circ} \mathrm{C}\right)$ & $X_{\mathrm{c}}(\%)$ \\
\hline HDPE & 303 & 2.5 & - & 134.3 & 73.6 \\
LLDPE & 145 & 2.0 & 13.2 & 119.3 & 35.0 \\
\hline
\end{tabular}

molecular weight distribution (MWD) were measured by gel permeation chromatography (GPC) using a PL GPC 210 operated at $160^{\circ} \mathrm{C}$ with dichlorobenzene as the eluent and at a flow rate of $10 \mathrm{~mL} \mathrm{~min}^{-1}$. The instrument was calibrated with polystyrene standards. The degree of short-chain branching (SCB) was calculated from ${ }^{13} \mathrm{C}$ NMR spectra recorded on a Bruker DMX-300 NMR spectrometer in 10\% solution of 1,2,4trichlorobenzene (TCB) at $125^{\circ} \mathrm{C}$. DSC was operated on a Mettler-Toledo DSC $822 \mathrm{e}$ with a heating rate of $10^{\circ} \mathrm{C} \mathrm{min}^{-1}$ in $\mathrm{N}_{2}$. The calculation of crystallinity $\left(X_{\mathrm{c}}\right)$ from DSC measurements was based on the heat of fusion of $273 \mathrm{~J} \mathrm{~g}^{-1}$ for the perfect polyethylene crystals.

Solutions with various ratios of linear and branched polyethylene mixtures were prepared by solving in xylene at $c a .130^{\circ} \mathrm{C}$, and the total concentration is $0.5 \mathrm{wt} \%$. The hot solution was dropped on a silica wafer inclined on a hot stage at about $130^{\circ} \mathrm{C}$. The inclination of the wafer made the polymer solution flow on it and formed a continuous film thin enough before the solvent evaporates completely under such temperature. After $10 \mathrm{~min}$, the solvent totally evaporated and the polymer was still in a molten state. Then the wafer was cooled down to room temperature at about $2{ }^{\circ} \mathrm{Cmin}^{-1}$, and the sample obtained was observed directly by AFM. The instrument used for accessing the morphology is Multimode Nanoscope IIIa (Digital Instruments). All images were obtained in ambient air with a tapping mode using silica pyramidal tips. 


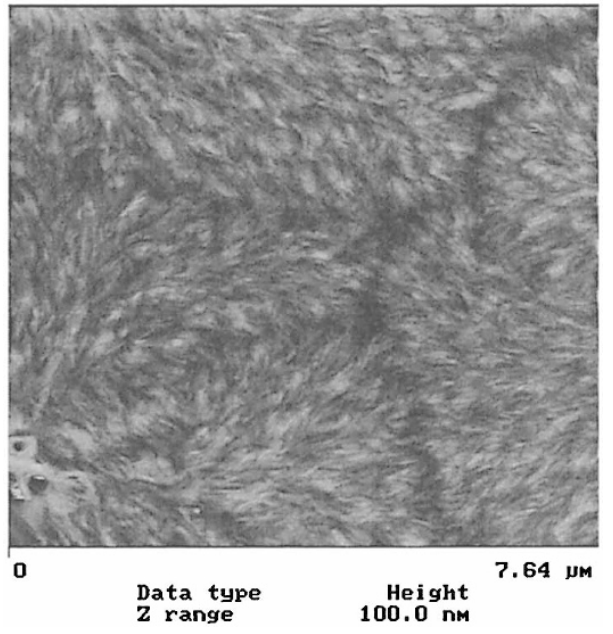

Figure 1. Tapping mode AFM image of the boundary region between spherulites of a commonly quenched HDPE thin film.

\section{RESULTS AND DISCUSSION}

Generally, under a suitable crystallization condition (e.g., temperature, pressure etc.), crystal nuclei will be created in a polymer melt firstly. All the lamellae growing from the same nucleus will form a spherulite. At last, the individual spherulites will collide with each other and the process of crystal growth primarily ends. The lamellae correlated with different spherulites just huddled together at the interface as shown in the AFM image of a commonly quenched HDPE of Figure 1.

For a dilute solution of crystallizable polymers, when the polymer concentration is low enough, the formed spherulites after casting and melt-crystallization will not be able to touch each other due to the using up of the macromolecules. Then what will the space between the spherulites be filled with? Figure 2a is AFM image of HDPE film prepared from such a dilute solution.

(a)

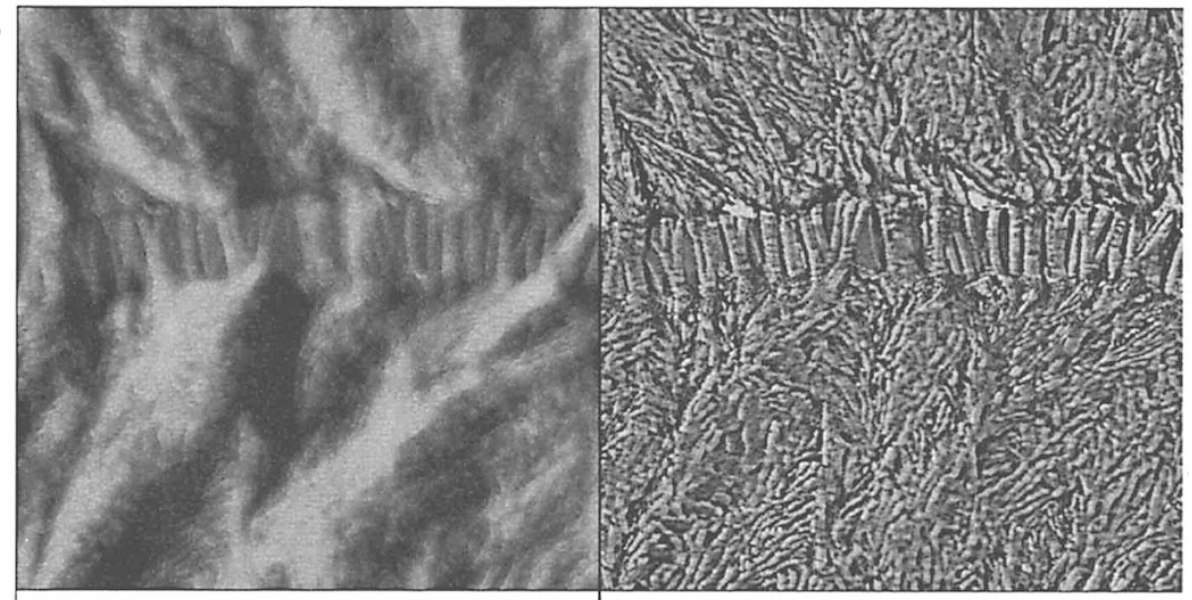

0

$3.91 \mathrm{\mu M} 0$

Data type
2 range

60.00 Phase

$3.91 \mathrm{\mu m}$

Data type Height
$100.0 \mathrm{~nm}$

(b)

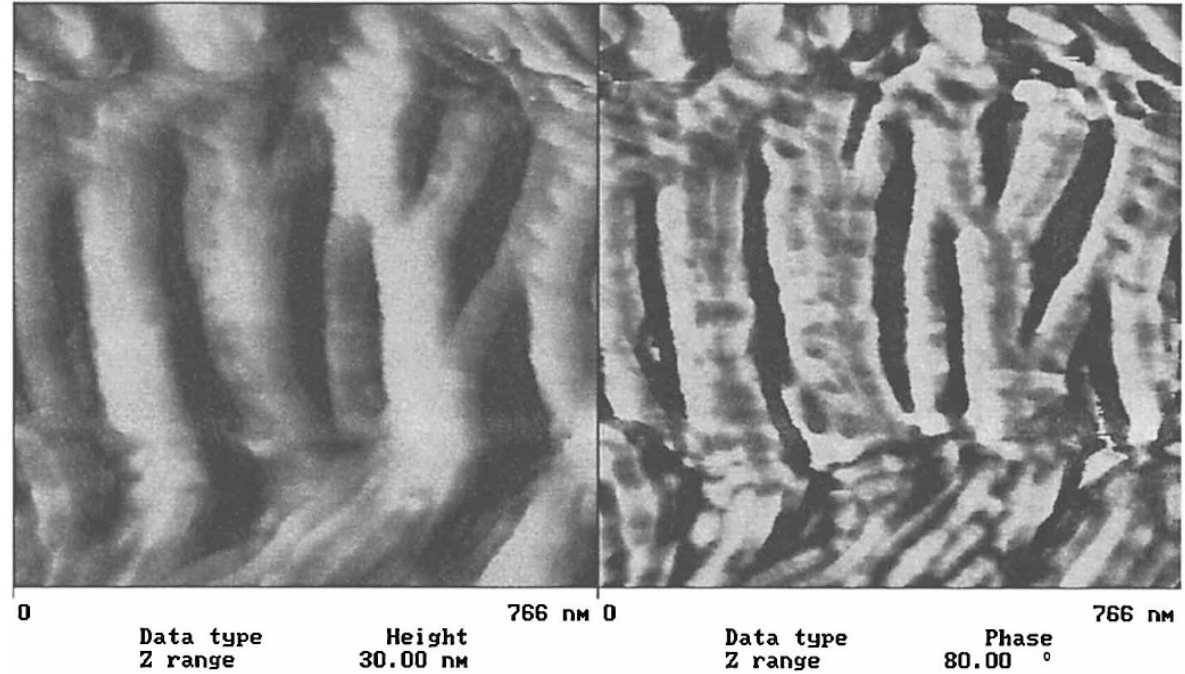

Figure 2. (a) Tapping mode AFM images of the boundary region between spherulites of a slowly cooled HDPE thin film. Left: height image, right: phase image. (b) The magnification of a portion of (a). 
It can be seen that there is a clear regular structure between spherulites and a lot of bundle-like bridges spanning the neighboring spherulites. All the spherulites seem to be in the same level of several tens nanometers in height image. According to the phase image, the regular structure is mainly crystalline. This is different from a result that the interface of spherulites is mainly filled with amorphous composition, which is believed to be pushed out in the crystallization process of the crystalline composition. The regular bundles are primarily parallel to each other and perpendicular to the border-line of the neighboring spherulites. There are also a few of branches in the inter- and intra-bundles of the regular structure. The magnified results supplied the detailed characteristics of the regular structure (Figure $2 \mathrm{~b}$ ), especially in the phase image. The bundle is about $500 \mathrm{~nm}$ long, $150 \mathrm{~nm}$ wide, and less than $20 \mathrm{~nm}$ high, being mainly made up of crystalline composition.

In the early literature, the tie molecules and interlamellar links were reported through dissolving away paraffin from its mixture with polyethylene. ${ }^{8,9}$ It was explained that parts of the same polyethylene (PE) molecules crystallized in more than one lamella. Though the sample used in the literature was prepared by melt mixing, the crystallization circumstance for $\mathrm{PE}$ was somewhat like that in solution due to the high molecular mobility of paraffin and the low concentration of polyethylene. While in present work, though the sample was prepared by solution casting, the intrinsic process is bulk crystallization in molten state. The spherulites obtained are the real bulk structure of polyethylene without other small molecules. As well known, the molecular motion behavior in bulk crystallization is absolutely different from that in solution crystallization.

We supposed that the formation of the regular structure is related to the force field caused by the stress in crystallization frontier between the neighboring spherulites, which shrink apparently during the meltcrystallization process. The slowly cooling process makes it possible to form compact structure and to concentrate the stress on the interface of the spherulites. Therefore, the formation mechanism of the regular structure should be similar to that of the fiber crystals caused by shear field, just the origins of the force fields are different. ${ }^{10,11}$ The composition of the regular structure should also be similar to the fiber crystals, i.e., the macromolecules in the regular region being basically parallel to each other. However, there is not the regular structure in the quenched sample with the same composition. The crystallization rate is so fast in quenched samples that stress caused by shrinkage in the melt-crystallization process was frozen in the bulk of polymer. There must be more defects and cavities in quenched polymer, agreeing with its general lower bulk density compared with that of slowly cooled polymer.

The more importance of the regular structure is that it supplies a model of crystallization process during the formation of polymeric spherulites. That is, there should be also a similar force field in the frontier of the crystalline region during the crystallization process, even at its very early initiation. In fact, the shrinking stress is related to the condensed and the molten states of polymers, the interaction between macromolecules, and the thermal motion of macromolecules. The competition between condensed and molten states for polymer molecules or segments varies with temperature, which should be the intrinsic factor affecting the crystallization kinetics. Also, it should be the shrinking stress that makes macromolecules order locally in radial direction and results in the microcosmic regularity in the formed spherulite. Since this is a fundamental problem in condensed macromolecular physics, no doubt the hypothesis needs to investigate widely and in detail.

Figure $3 \mathrm{a}$ is AFM image of the blend of $80 \%$ linear and $20 \%$ branched PE. The regular structure is also very clear and has the similar characteristics as that in HDPE. The difference is that there are some striations perpendicular to the length of the regular bundle. The magnified result illuminated that it was somewhat like the structure of shish-kebab crystals (Figure $3 b) .{ }^{12}$ It is not difficult to understand because of the same reason as for HDPE. The branched composition decreased the crystallization ability of the whole polymeric blend. The shorter molecules of the linear composition and the shorter segments of the branched composition will crystallize epitaxially on the outer surface of the fiber crystals formed by the longer linear macromolecules in the center of the regular bundle. The lamellae in spherulites became discontinuous and seemed like some regularly ranged bangers. This should also be related to the decreased crystallization ability of the whole blend due to the addition of the branched component.

When the branched component increased further, the regular structure between spherulites disappeared. It turned back to the common characteristics of the general polymer spherulites, just as the crystallization ability became even worse. Obviously, the decrease of linear composition made it difficult to form sufficient regular structure. Also, the increase of branched composition made it difficult to accumulate the shrinking stress in crystalline frontier due to the same reason that macromolecules are easy to relax in solution. 
(a)

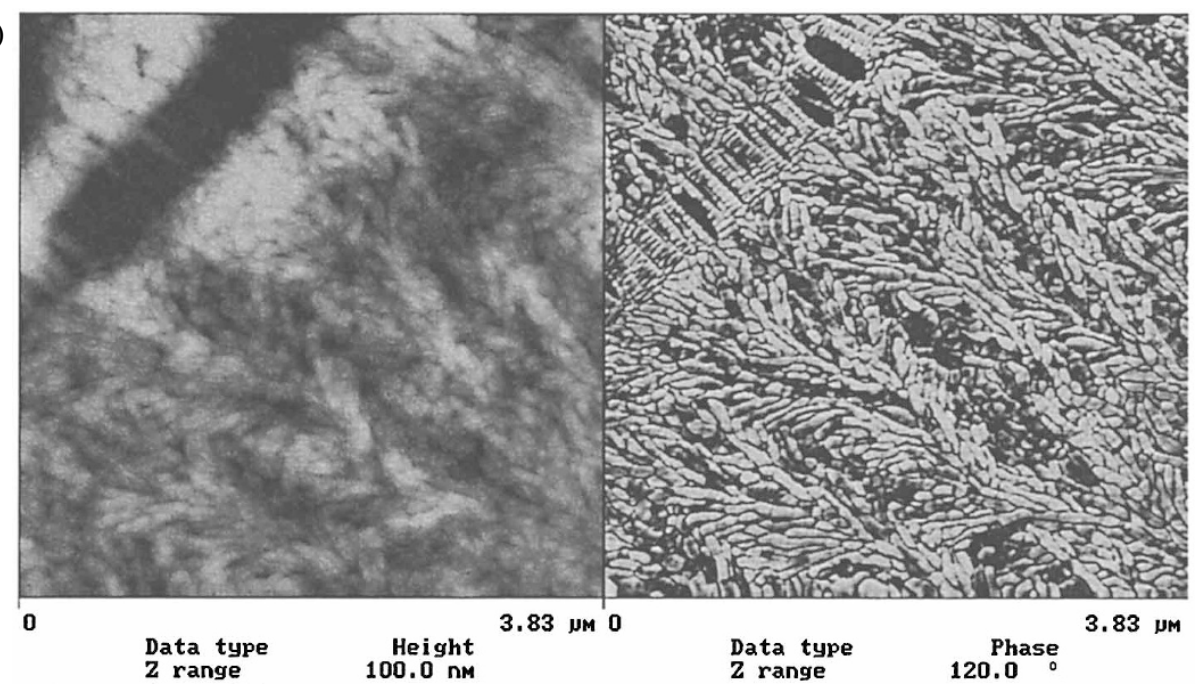

(b)

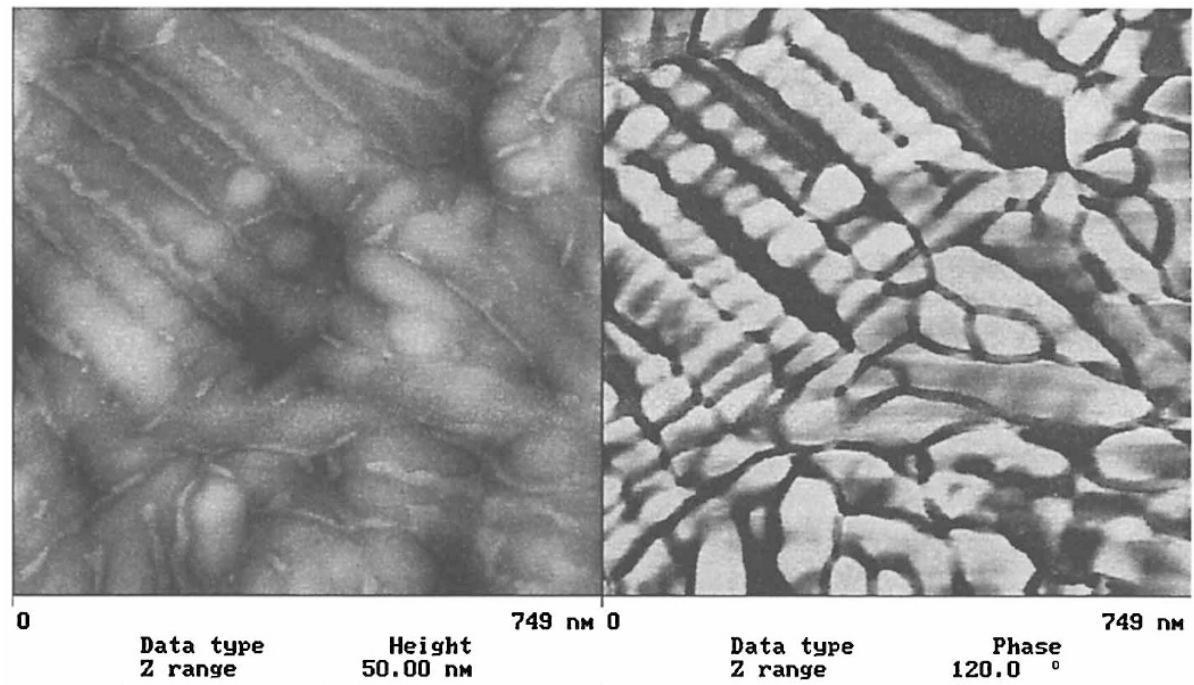

Figure 3. (a) Tapping mode AFM images of the boundary region between spherulites of an $80 \%$ linear and $20 \%$ branched PE blend thin film. Left: height image, right: phase image. (b) The magnification of a portion of (a).

Acknowledgment. This work was supported by the National Science Foundation of China (20004011 and 20023003).

\section{REFERENCES}

1. N. H. Hartshorne and A. Stuart, "Crystals and the Polarizing Microscope", Edward Arnold Publishers Ltd., London, 1970.

2. R. A. Chivers, S. J. Martinez, P. J. Barham, and A. Keller, J. Polym. Sci., Polym. Phys. Ed., 20, 1717 (1982).

3. L. C. Sawyer and D. T. Grubb, "Polymer Microscopy", Chapman and Hall Ltd., New York, N.Y., 1987.

4. M. J. Hill and P. J. Barham, Polymer, 33, 4099 (1992).

5. L. Reimer, 'Electron Spectroscopic Imaging' in "EnergyFiltering Transmission Electron Microscopy” L. Reimer, Ed.,
Springer-Verlag, GmbH \& Co., Berlin, 1984, p 347.

6. H. Tadokoro, "Structure of Crystalline Polymers", John Wiley \& Sons, Inc., New York, N.Y., 1979.

7. J. A. Chandler, "X-ray Microanalysis in the Electron Microscope", North-Holland Publishers, Amsterdam, 1977.

8. H. D. Keith, F. J. Padden, and R. G. Vadimsky, Science, 150, 1026 (1965).

9. H. D. Keith, F. J. Padden, and R. G. Vadimsky, J. Polym. Sci., A-2, 4, 267 (1966).

10. A. Zwijnenburg and A. J. Pennings, Colloid Polym. Sci., 253, 452 (1975).

11. A. J. Pennings, R. Lagaveen, and R. S. Devries, Colloid Polym. Sci., 255, 532 (1977).

12. P. J. Barham, Polymer, 23, 1112 (1982). 